\title{
Person-Centered Care in a Tertiary Hospital Through Patient's Eyes: A Cross-Sectional Study
}

This article was published in the following Dove Press journal:

Patient Preference and Adherence

\author{
Bashayer Al-Sahli ${ }^{1,2}$ \\ Abdelmoneim Eldali ${ }^{1}$ \\ Mohammed Aljuaid (iD ${ }^{3}$ \\ Khaled Al-Surimi (iD) $2,4,5$ \\ 'King Faisal Specialist Hospital and \\ Research Center, Riyadh, Saudi Arabia; \\ ${ }^{2}$ Department of Health System \\ Management, College of Public Health \\ and Health Informatics, King Saud Bin \\ Abdulaziz University for Health Sciences, \\ Riyadh, Saudi Arabia; ${ }^{3}$ Department of \\ Health Administration, King Saud \\ University, Riyadh, Saudi Arabia; ${ }^{4}$ King \\ Abdullah International Medical Research \\ Center, King Saud Bin Abdulaziz \\ University for Health Sciences, Riyadh, \\ Saudi Arabia; ${ }^{5}$ Community Medicine \\ Department, Faculty of Medicine \& \\ Health Sciences, Thamar University, \\ Dhamar, Yemen
}

Background: The current trend in healthcare systems around the world is moving towards a person (or patient)-centered care (PCC) model. While some healthcare organizations have only recently embraced the PCC approach, there is no evidence of person-centered care in Saudi hospitals. This study aimed to assess patients' perspectives on the climate of personcentered care and its associated factors in a tertiary hospital in Saudi Arabia.

Methods: A cross-sectional study design was conducted in 16 inpatient departments at a tertiary hospital in Riyadh, Saudi Arabia. The validated version of the Person-centered Climate Questionnaire-Patient (PCQ-P) was distributed to 300 adult patients admitted to the hospital for more than 48 hours. The patients were interviewed face-to-face, using the PCQP. Descriptive and inferential statistical analysis were performed using SPSS (version 22; IBM, NY, USA).

Results: A total of 300 questionnaires were completed. More than half (53\%) were females; $39.7 \%$ aged $21-40$ years; $72.7 \%$ married; $49 \%$ hold a high school diploma; and $67.4 \%$ unemployed. For route of admission into hospital, 39.8\% of the respondents were admitted as elective or outpatient appointments, and $45 \%$ had been admitted for less than a week. Most patients $(84.3 \%)$ reported that they preferred to be treated in a governmental hospital. Inpatients' overall mean PCQ-P score was $73 \pm 9.988$ out of 85 . Results suggested significant associations between patient characteristics and their perspectives on person-centered care, such as age $(P=0.005)$, gender $(P<0.001)$, nationality $(P=0.026)$, area of residency $(P=0.001)$, route to admission $(P=0.002)$, length of stay $(P=0.003)$, and hospital preference $(P=0.010)$.

Conclusion: Overall, patients' perspective on person-centered care seems positive. Patient and hospital characteristics could play an important role in shaping patients' perceptions of the climate of person-centered care domains.

Keywords: person-centered care, patients' perspective, hospital, PCQ-P - Arabic version

\section{Background}

Many healthcare systems have gradually shifted toward person-centered care approach. In the report entitled "Crossing the quality chasm", the Institute of Medicine (IOM) drew up six proposals reinforcing the notion that healthcare should be safe, effective, person-centered, timely, efficient, and equitable. ${ }^{1}$ Recently, McCormack et al proposed the following definition:

Person-centeredness is an approach to practice established through the formation and fostering of healthful relationships between all care providers, service users and others significant to them in their lives. It is underpinned the values of respect for persons (personhood), individual right to self-determination, mutual respect and understanding. It
Correspondence: Khaled Al-Surimi Department of Health System

Management, College of Public Health and Health Informatics, King Saud Bin Abdulaziz University for Health Sciences, P.O. Box 3660, Riyadh, Saudi Arabia Email kalsurimi@gmail.com 
is enabled by cultures of empowerment that foster continuous approaches to practice development. ${ }^{2}$

This definition focuses on the importance of relationships that are built on mutual respect and understanding between the patients, their family members and healthcare professionals. The term "patient-centered care" is often used interchangeably with the term "person-centered care" in the literature. ${ }^{3}$

Ekman et al highlighted that term "patient" is mostly used as a passive and weak receiver of care, while the term "person" is a core partner that participate in the team providing the care. ${ }^{4}$ In addition, person-centered care is different than patient-centered care in considering the whole person not only their medical conditions as a patient, and allowing the person to play active role by involving in healthcare process and decision making while the concept of "patient" limiting the person to more inactive role. ${ }^{5,6}$ Furthermore, the term "person" involves patients, their families and health care staff as equal partners in planning and developing the best way of providing healthcare.

In summary, the PCC model differs from the traditional biomedical/healthcare model in its goal of involving patients in the decision-making process and taking the responsibility for their decisions under the healthcare provider's supervision. ${ }^{7}$ The traditional healthcare model focuses mainly on the disease, and the healthcare provider's key role in making all relevant and necessary clinical decisions to manage and cure the disease through different treatment options and interventions. ${ }^{7}$

Several studies have attempted to identify the benefits of applying the PCC model in healthcare. In a study involving hip or knee replacement patients, those who underwent surgery within the patient and family-centered care program, had a high level of patient satisfaction and functional status, a low infection rate, and a shorter length of hospital stay. ${ }^{8}$ Other studies have also reported a significant relationship between PCC and decreased mortality, emergency return visits, and medication errors. ${ }^{9,10}$ A recent study in a psychosis care setting resulted in increased patient satisfaction post a PCC intervention for patients with schizophrenia spectrum disorders. ${ }^{11}$

The available evidence supports the positive impact of PCC on health outcomes. ${ }^{12,13}$ Moreover, several studies have shown that person-centered approaches can increase patientdoctor satisfaction, engagement, task orientation, reduce anxiety, and improve quality of life. There is also some evidence to suggest that PCC is highly efficient, resulting in fewer diagnostic tests and unnecessary referrals. ${ }^{14}$
The common core values of PCC are respect for patients' values and preferences, coordination and integration of care, emotional support, physical comfort, information, communication, health education, continuity and transition, the involvement of family and friends, and access to care. ${ }^{7,9,15}$ A survey of patient preferences found that patients rank communication, shared decision-making, and health promotion as their most essential needs. ${ }^{7}$ Another recent qualitative study identified three necessities that patients' needs during cancer treatment: (1) psychosocial and supportive care needs, (2) information needs, such as, education, information on complementary therapy and financial cost and support and (3) quality of information delivery where patient expressed their needs for more time from professionals and simple language. ${ }^{16}$

In Saudi Arabia, the healthcare system allows patients free access to government-provided healthcare services, ${ }^{17}$ and the quality of healthcare at all levels has markedly improved in recent years. Nevertheless, concerns about the quality of care and patient safety are increasing. ${ }^{18}$ In other words, it remains a challenge for Saudi health organizations to obtain patients' views on the quality of care and the extent of their involvement. There is also a general tendency towards depicting view patients as passive receivers of care rather than being in a partnership with the healthcare providers, and sharing their healthcare choices and plans. ${ }^{19}$ A study conducted in a governmental hospital in Saudi Arabia revealed that $63.4 \%$ of participants were unaware of their right to be kept fully informed of their diagnosis and treatment plan. ${ }^{17}$

It seems that the health system in Saudi Arabia was captured as a resource and staff centric rather than patient or person centric. However, the Ministry of Health (MoH) has identified patients involvement and views issue as one of the major challenges that need to be undertaken in the future [20 February]. ${ }^{20}$ Consequently, Saudi Arabia proposed transformational goals for the vision 2030 that are committed to: improve health and quality of life, improve healthcare and accountability of healthcare organizations and staff to deliver care that is safe, effective, patientcentered, timely, and equitable; and improve value of care by containing costs and improving outcomes. ${ }^{21}$

While the PCC approach has been widely adopted across different healthcare settings worldwide, it is still relatively new to some health organizations in Saudi Arabia, and there have been few studies to measure PCC from patient's perspective. ${ }^{22,23}$ Therefore, it is vital to evaluate and promote a person-centered approach that 
help improve health outcomes and reduce care costs in Saudi hospitals. Thus, the aims of this study were to assess patients' perspectives the person-centered care climate in a Saudi tertiary hospital, and identify any significant associations between their perspectives and characteristics.

\section{Methods}

\section{Study Design and Setting}

This cross-sectional study was conducted at a tertiary hospital in Riyadh, Saudi Arabia. The sample size was calculated using $\mathrm{G}^{*}$ power 3.0 software. ${ }^{24}$ Using a power level of 0.80 , an alpha level of 0.05 , and with the medium effect size of 0.30 for a two-tailed independent $t$-test, the minimum estimated sample size was 134 . The sample size was then increased to 300 participants to ensure that a large sample is obtained providing more valid and reliable findings. The inclusion criteria for participants were adult patients admitted to the hospital for more than 48 hours to in one of the 16 inpatient departments, including General Surgery; Medical and Surgical Cardiology; Urology; Ear, Nose and, Throat; Gynecology; Hematology, and Internal Medicine over a seven month period in 2017. All patients were voluntarily willing to participate in the study. Pediatric patients and patients deemed unstable or unconscious were excluded.

\section{Data Collection}

The PCQ-P, which was first introduced by researchers in Sweden to measure patients' perspectives on the care environment in a healthcare setting, comprises 17 items, covering three dimensions of the person-centered care climate: Safety, Everydayness, and Hospitality. ${ }^{25}$ The PCQ-P was translated into English and validated by Australian study. ${ }^{25}$ The Cronbach's alpha value for the original Swedish version was 0.93 on total scale, with values of $0.94,0.82$ and 0.64 for the three subscales of safety, everydayness, and hospitality, respectively. ${ }^{26}$ For the purpose of our study, using forward-backward method, English version of PCQ-P was translated from English into Arabic by a professional translator and modified to ensure reliability and clarity based on feedback from a small pilot study of 20 participants. Following the pilot study, we reviewed the content of the PCQ-P to ensure that it was appropriate for the Saudi cultural context. The original tool used in this research was translated previously to different languages using the same method. ${ }^{27}$ Unlike the original questionnaire, which uses a 6-point Likert scale, a 5-point scale, ranging from 1 (No, I disagree completely) to 5 (Yes, I agree completely) was opted for. Total PCC climate scores range from 17 , indicating a not very personcentered climate, to 85 , a very person-centered climate.

A trained nurse, who was not working at any of the 16 different wards mentioned above, conducted face-to-face interviews with a proportional sample of patients meeting the inclusion criteria. During the interviews, participants were asked the questions from the Person-Centered Climate Questionnaire - Patient (PCQ-P) version. Data related to respondents' characteristics, including age, gender, marital status, health insurance status, education level, occupation, income, nationality, length of hospital stay, route of hospital admission, area of residency and treatment preferences, were added to the PCQ-P questionnaire, and collected during interviews.

\section{Statistical Analysis}

All statistical analyses were performed using SPSS (version 22; IBM, NY, USA). Data were summarized using mean $\pm \mathrm{SD}$ and/or frequencies and percentages, as appropriate. Furthermore, t-tests and analysis of variance (ANOVA) were used to compare the means between various groups. Univariate and multivariate regression analyses were used to identify significant independent factors associated with PCQ-P score. The means of Safety, Everydayness, Hospitality and Overall scores were used to define cut-off points. The significant level was set after applying the Holm-Bonferroni correction at $P<0.05$. Effect size was calculated to quantify the difference between two groups. ${ }^{28}$ Finally, regression analyses were performed to identify the significant predictors of person-centred care domains.

\section{Results}

\section{Respondents' Characteristics}

A total of 300 questionnaires were completed. More than half of the respondents (53\%) were females; 39.7\% aged 21-40 years; $72.7 \%$ married; $49 \%$ had attained up to a high school level of education; and $67.4 \%$ unemployed. One-third $(29.7 \%)$ of respondents reported that they had no income, approximately one-fourth (24.7\%) earned more than 10,000 SAR per month. Most participants (96.7\%) were of Saudi nationality; and $60.7 \%$ lived outside Riyadh. The proportion of participants with no health insurance was $85.3 \%$. As for route of admission into hospital, $39.8 \%$ of respondents were admitted as elective or outpatient 
appointments, and $45 \%$ had been admitted for less than a week. Most patients (84.3\%) reported that they preferred to be treated in a governmental hospital (Table 1).

\section{Person-Centered Care Climate}

The overall mean score for the PCQ-P was $73 \pm 9.988$ out of 85 , indicating that the participants in this study perceived their healthcare environments as a highly person-centered climate of care. The highest two mean scores for the PCQ-P were achieved by item 3, "A place where I feel safe" (4.95 \pm $0.39 \mathrm{SD}$ ), and item 9, "A place that is neat and clean" (4.90 $\pm 0.54 \mathrm{SD}$ ); both of which are related to Safety. The item with the lowest mean score was item 11, "A place that has something nice to look at (eg, views, or artwork, etc.)" (2.51 $\pm 1.82 \mathrm{SD}$ ) pertaining to Everydayness (see Table 2).

For Hospitality, the highest two mean scores were achieved by item 1, "A place where the staff are knowledgeable" (4.89 $\pm 0.59 \mathrm{SD})$, and item 4, "A place where I feel welcome" (4.89 $\pm 0.54 \mathrm{SD})$. However, patients positively agreed with all of the Hospitality domain items; percentage scores ranged from $77.7 \%$ to $96 \%$, indicating that patients perceived a good climate of hospitality in the hospital. As mentioned earlier, the statement receiving the highest mean score in the domain of Safety - and the PCQ-P overall - was item 3, while, the lowest mean score in the Safety domain was item 10, "A place where the staff seem to have time for patients, $(4.36 \pm 1.38 \mathrm{SD})$. The percentage scores in this domain ranged from $81 \%$ to $98 \%$, indicating a very high perception of a climate of safety in the hospital. The highest mean score in the domain of Everydayness was achieved by item 12, "A place that feels homely" (3.96 $\pm 1.67 \mathrm{SD})$; whereas, the lowest scoring item in this domain - and in the PCQ-P overall - was item 11. Percentage scores of agreements in this domain ranged from $32.4 \%$ to $70.5 \%$, revealing a low patient perception of a climate of everydayness. Table 3 shows more details about correlation coefficient between PCC and respondents' characteristics.

\section{Predictors of and Factors Associated with the Climate of Person-Centered Care}

Associations between the mean overall PCQ-P scores, Safety, Hospitality, and Everydayness, and independent variables were analyzed by t-tests and ANOVA and presented in Table 4. The overall PCQ-P scale had a maximum score of 85, divided between Hospitality (40), Safety (25), and
Table I Respondents' Characteristics

\begin{tabular}{|c|c|c|}
\hline Variables & No & $\%$ \\
\hline \multicolumn{3}{|l|}{ Age (years) } \\
\hline$\leq 20$ & 20.0 & 6.7 \\
\hline $21-40$ & 119.0 & 39.7 \\
\hline $4 I-60$ & 102.0 & 34.0 \\
\hline$\geq 60$ & 59.0 & 19.7 \\
\hline \multicolumn{3}{|l|}{ Gender } \\
\hline Male & $|4|$ & 47 \\
\hline Female & 159 & 53 \\
\hline \multicolumn{3}{|l|}{ Nationality } \\
\hline Saudi & 290.0 & 96.7 \\
\hline Non-Saudi & 10.0 & 3.3 \\
\hline \multicolumn{3}{|l|}{ Health insurance } \\
\hline Yes & 44.0 & 14.7 \\
\hline No & 255.0 & 85.3 \\
\hline \multicolumn{3}{|l|}{ Marital status } \\
\hline Married & 218.0 & 72.7 \\
\hline Unmarried & 82.0 & 27.3 \\
\hline \multicolumn{3}{|l|}{ Educational level } \\
\hline Illiterate & 47.0 & 15.7 \\
\hline High school and below & 147.0 & 49.0 \\
\hline Bachelor & 96.0 & 32.0 \\
\hline Postgraduate & 10.0 & 3.3 \\
\hline \multicolumn{3}{|l|}{ Occupation } \\
\hline Employed & 97.0 & 32.6 \\
\hline Unemployed & 201.0 & 67.4 \\
\hline \multicolumn{3}{|l|}{ Monthly income (SR) } \\
\hline No income & 88.0 & 29.7 \\
\hline$<5000$ & 61.0 & 20.6 \\
\hline $5000-10,000$ & 74.0 & 25.0 \\
\hline$>10,000$ & 73.0 & 24.7 \\
\hline \multicolumn{3}{|l|}{ Area of residency } \\
\hline Riyadh & 118.0 & 39.3 \\
\hline Outside Riyadh & 182.0 & 60.7 \\
\hline \multicolumn{3}{|l|}{ Area of admission } \\
\hline Emergency department & 91.0 & 30.4 \\
\hline Elective case or outpatient appointment & 119.0 & 39.8 \\
\hline Referral from other hospital & 89.0 & 29.8 \\
\hline \multicolumn{3}{|l|}{ Duration of hospitalization (weeks) } \\
\hline$<1$ & 135.0 & 45.0 \\
\hline $\mathrm{I}-2$ & 79.0 & 26.3 \\
\hline$>2$ & 86.0 & 28.7 \\
\hline \multicolumn{3}{|l|}{ Hospital preference for treatment } \\
\hline Governmental & 252.0 & 84.3 \\
\hline Teaching & 6.0 & 2.0 \\
\hline Private & 41.0 & 13.7 \\
\hline
\end{tabular}


Table 2 Patient-Centered Climate Questionnaire - Patient (PCQ-P) Version: Mean Score and Standard Deviation

\begin{tabular}{|c|c|}
\hline PCQ-P/Item & Mean \pm SD \\
\hline \multicolumn{2}{|l|}{ Hospitality (Cronbach's alpha: 0.766 ) } \\
\hline I. A place where staff are knowledgeable & $4.76 \pm 0.59$ \\
\hline 4. A place where I feel welcome & $4.84 \pm 0.52$ \\
\hline 5. A place where it is easy to talk to staff & $4.56 \pm 0.94$ \\
\hline 6. A place where staff take notice of what I say & $4.67 \pm 0.80$ \\
\hline $\begin{array}{l}\text { 8. A place where staff talk to me so that I can } \\
\text { understand }\end{array}$ & $4.78 \pm 0.60$ \\
\hline $\begin{array}{l}\text { 15. A place where staff make extra efforts for my } \\
\text { comfort }\end{array}$ & $4.18 \pm 1.33$ \\
\hline $\begin{array}{l}\text { 16. A place where I can make choices (eg what to } \\
\text { wear, eat, etc.) }\end{array}$ & $4.60 \pm 0.93$ \\
\hline 17. A place where I can get that "little bit extra" & $4.49 \pm 1.01$ \\
\hline \multicolumn{2}{|l|}{ Safety (Cronbach's alpha: $\mathbf{0 . 6 9 0 )}$} \\
\hline 2. A place where I receive the best possible care & $4.61 \pm 0.89$ \\
\hline 3. A place where I feel safe & $4.92 \pm 0.40$ \\
\hline $\begin{array}{l}\text { 7. A place where staff come quickly when I need } \\
\text { them }\end{array}$ & $4.34 \pm 1.13$ \\
\hline 9. A place that is neat and clean & $4.86 \pm 0.55$ \\
\hline $\begin{array}{l}\text { 10. A place where staff seem to have time for } \\
\text { patients }\end{array}$ & $4.33 \pm 1.16$ \\
\hline \multicolumn{2}{|l|}{ Everydayness (Cronbach's alpha: 0.684 ) } \\
\hline $\begin{array}{l}\text { II. A place that has something nice to look at (eg } \\
\text { views, artwork, etc.) }\end{array}$ & $2.55 \pm 1.64$ \\
\hline 12. A place that feels homely & $3.89 \pm 1.50$ \\
\hline $\begin{array}{l}\text { 13. A place where it is possible to get unpleasant } \\
\text { thoughts out of your head }\end{array}$ & $3.67 \pm 1.63$ \\
\hline $\begin{array}{l}\text { 14. A place where people talk about everyday life } \\
\text { and not just illness }\end{array}$ & $2.96 \pm 1.77$ \\
\hline
\end{tabular}

Everydayness (20). In terms of the overall PCQ-P, the only independent variables found to have a significant association were age (patients younger than 20 years, $P=0.005$ ), gender (males, $P<0.001$ ), nationality (Saudis, $P=0.026$ ), area of residency (living outside Riyadh, $P=0.001$ ), route of admission (referred from another hospital, $P=0.002$ ), length of stay (more than two weeks, $P=0.003$ ), and hospital preference for treatment (governmental hospitals, $P=0.010$ ).
The following independent variables were found to be significantly associated with the Hospitality dimension: age (41-60 years; $P<0.001$ ), gender (male, $P<0.001$ ), employment status (employed, $P=0.002$ ), area of residency (living outside Riyadh, $P=0.019$ ), and length of stay (more than two weeks, $P=0.052$ ). As for the Safety dimension, the following were significantly associated: age (20 years and younger, $P=0.012$ ), gender (male, $P=0.000$ ), employment status (employed, $P=0.001$ ), area of residency (living outside Riyadh, $P=0.000$ ), length of stay (more than 2 weeks, $P=0.005$ ), and hospital preference (governmental hospitals, $P=0.016$ ).

For Everydayness, significant associations were found with gender (males, $P=0.001$ ), area of residency (living outside Riyadh, $P=0.001$ ), route of admission (referred from another hospital, $\mathrm{P}=0.002$ ), length of stay (more than 2 weeks, $P=0.015$ ), and hospital preference (governmental hospitals, $P=0.032$ ).

Multivariate linear regression analysis was used to identify significant predictors of PCC, both as a whole and at the level of the three subscales. Multivariate analysis showed that male perceived higher PCC than female and was significantly associated with Everydayness ( $P=0.004$ ), while age of 41-60 was significantly associated with Hospitality $(P=0.009)$. Living outside Riyadh was a significant predictor of $\mathrm{PCC}$ in the Hospitality dimension ( $P=0.040)$, and in the PCQ-P overall $(P=0.047)$. Length of hospitalization: patient, who stayed more than two weeks in the hospital, was a significant predictor of all scales (Hospitality, $P=0.010$; Safety, $P<0.001$; Everydayness, $P=0.007$; overall, $P=0.001$ ). As for preference of hospital type for treatment, the public hospital was only significantly associated with patients' perceptions of the hospital environment as being person-centered in terms of Safety dimension and the overall PCC scale (see Table 5).

\section{Discussion}

The primary aim of this study was to assess and provide empirical evidence of patients' perceptions of the climate of person-centered care (PCC) at a tertiary care hospital in Saudi Arabia. Understanding these perceptions will, in turn, help healthcare providers and policymakers rethink the extent to which the PCC model is used in Saudi hospitals. The study also explored the relationships between inpatient characteristics and their perceptions of the climate of PCC domains. The PCC climate mainly 


\begin{tabular}{|c|c|c|c|c|c|c|c|c|c|c|c|c|c|c|}
\hline 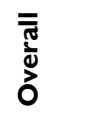 & 员 & 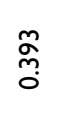 & $\frac{\stackrel{*}{*}}{\frac{*}{\Lambda}}$ & $\stackrel{m}{0}$ & $\begin{array}{l}\text { on } \\
0 \\
0\end{array}$ & $\frac{f}{0}$ & $\begin{array}{l}\text { ڤ̊ } \\
\text { }\end{array}$ & ô & 行 & 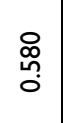 & $\begin{array}{l}\text { oे } \\
\text { i. }\end{array}$ & ỡ & $\begin{array}{l}\infty \\
0 \\
0\end{array}$ & $\begin{array}{l}\circ \\
\text { o̊ } \\
0\end{array}$ \\
\hline 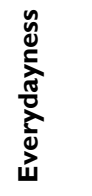 & $\begin{array}{l}0 \\
0 \\
0 \\
0\end{array}$ & $\stackrel{\stackrel{\infty}{\infty}}{\stackrel{0}{0}}$ & $\frac{\text { 善 }}{\frac{0}{0}}$ & $\bar{o}$ & $\begin{array}{l}\hat{\tilde{o}} \\
\text { } \\
\text { io }\end{array}$ & 志 & $\begin{array}{l}\infty \\
0 \\
0\end{array}$ & $\begin{array}{l}\stackrel{0}{0} \\
\text { O. } \\
0\end{array}$ & $\begin{array}{l}\text { Pơ } \\
0 \\
0\end{array}$ & 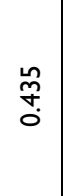 & $\begin{array}{l}\text { o } \\
0 \\
0\end{array}$ & $\frac{\bar{\Phi}}{0}$ & $\begin{array}{l}\text { 岁 } \\
\text { O. } \\
\text { i }\end{array}$ & 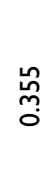 \\
\hline 离 & 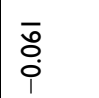 & 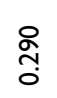 & $\frac{\stackrel{*}{\tilde{m}}}{0}$ & $\begin{array}{c}\tilde{O} \\
\stackrel{0}{0}\end{array}$ & $\begin{array}{l}\stackrel{0}{0} \\
\stackrel{0}{0}\end{array}$ & 운 & $\begin{array}{l}\frac{m}{0} \\
\dot{i}\end{array}$ & 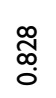 & $\begin{array}{l}\text { Z⿱ } \\
0 \\
0\end{array}$ & \begin{tabular}{l}
$\infty$ \\
\multirow{+}{*}{} \\
0
\end{tabular} & $\begin{array}{l}\stackrel{+}{0} \\
\stackrel{0}{0} \\
\stackrel{\varphi}{0}\end{array}$ & $\frac{\hat{y}}{0}$ & $\begin{array}{l}\hat{\mathrm{o}} \\
0 \\
0\end{array}$ & $\frac{\dot{m}}{0}$ \\
\hline 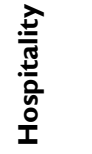 & $\begin{array}{l}0 \\
0 \\
0 \\
0 \\
0\end{array}$ & $\frac{\mathbb{Z}}{0}$ & 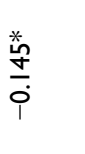 & $\frac{\mathrm{N}}{0}$ & $\begin{array}{l}\text { 芯 } \\
0 \\
0\end{array}$ & $\begin{array}{l}\text { o } \\
\text { h⿱⺈ } \\
0\end{array}$ & ஜ̣̊̊ & 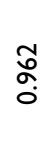 & đ̛̣ & $\begin{array}{l}\hat{o} \\
\text { fo } \\
0\end{array}$ & $\begin{array}{l}\text { ळ. } \\
0 \\
0\end{array}$ & $\frac{\stackrel{a}{m}}{0}$ & $\begin{array}{l}\text { Na } \\
\text { O. }\end{array}$ & $\frac{\widehat{N}}{O}$ \\
\hline $\begin{array}{l}\stackrel{\Xi}{E} \\
\stackrel{\Xi}{\underline{\Xi}}\end{array}$ & 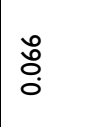 & 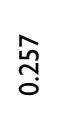 & 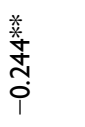 & ষ্ণ & oे & $\underset{\substack{\infty \\
\infty \\
0}}{\infty}$ & 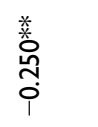 & ষ্ণ & 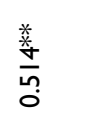 & ঃ & 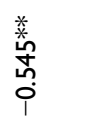 & ষ্ণ & $\underset{\text { ¿ }}{\text {. }}$ & \\
\hline 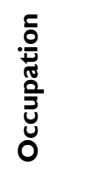 & 艿 & 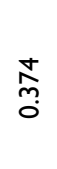 & $\frac{\stackrel{*}{m}}{m}$ & ষ্ণ & $\frac{\frac{*}{2}}{\frac{0}{i}}$ & ô & 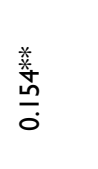 & 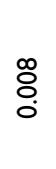 & 美 & ০০ & $\underset{\varnothing}{\stackrel{0}{0}}$ & & 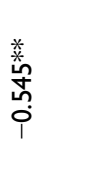 & : \\
\hline لَّ & 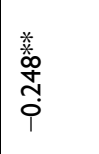 & ঃ̊ & 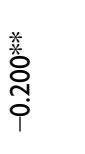 & : & ō & $\frac{o}{0}$ & 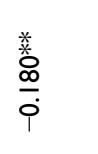 & రి & ه̊ & & 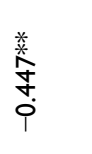 & ষ̊․ & 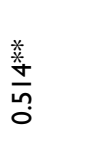 & 웅 \\
\hline 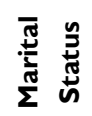 & 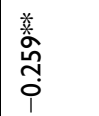 & ষ্ণ & o̊ & $\begin{array}{l}\bar{\alpha} \\
\text { o. }\end{array}$ & $\begin{array}{l}\stackrel{\infty}{0} \\
\stackrel{0}{i}\end{array}$ & $\begin{array}{l}\widetilde{3} \\
0 \\
0\end{array}$ & $\underset{-}{\text { O̦ }}$ & & $\frac{\frac{*}{0}}{0}$ & ర్ & 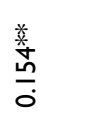 & $\stackrel{\circ}{\circ}$ & 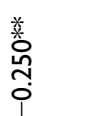 & 웅 \\
\hline 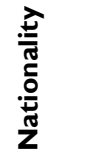 & $\begin{array}{l}n \\
\hat{o} \\
0 \\
0\end{array}$ & $\frac{\alpha}{0}$ & 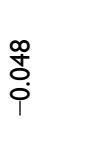 & 菑 & ه্ర & & $\begin{array}{l}\infty \\
\stackrel{\infty}{0} \\
0\end{array}$ & $\begin{array}{l}\widetilde{0} \\
\stackrel{0}{0}\end{array}$ & סूo & $\frac{o}{0}$ & $\frac{\frac{*}{2}}{i}$ & 㐱. & ठ̊. & $\underset{\substack{\infty \\
\infty \\
0 \\
0}}{0}$ \\
\hline ڤึ & $\begin{array}{l}\text { ¿̊ } \\
0 \\
0\end{array}$ & $\begin{array}{l}\hat{\hat{O}} \\
0 \\
0\end{array}$ & $\stackrel{8}{\circ}$ & & $\begin{array}{l}\infty \\
\stackrel{0}{0} \\
\stackrel{\varphi}{i}\end{array}$ & $\begin{array}{l}\text { to } \\
\vdots \\
0\end{array}$ & $\stackrel{\tilde{o}}{0}$ & $\begin{array}{l}\bar{\alpha} \\
\text { 。 }\end{array}$ & 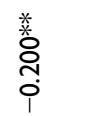 & 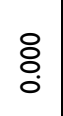 & $\frac{\stackrel{\text { w }}{\frac{m}{m}}}{\stackrel{m}{0}}$ & ঃ & $\begin{array}{l}\text { 尊 } \\
\stackrel{+}{\sim} \\
\text { i }\end{array}$ & ষ্ণ \\
\hline$\underset{\text { 总 }}{2}$ & ○̊․ & & 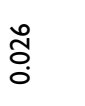 & 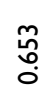 & $\begin{array}{l}\hat{\kappa} \\
0 \\
0\end{array}$ & $\frac{\alpha}{0}$ & 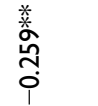 & ঃ & 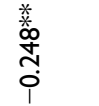 & $\stackrel{\circ}{\circ}$ & $\begin{array}{l}\text { గ్ } \\
\text { O̊ }\end{array}$ & 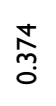 & 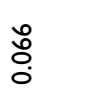 & ڤ્ત \\
\hline & 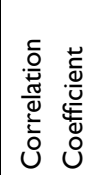 & 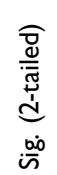 & 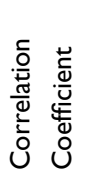 & 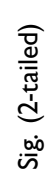 & 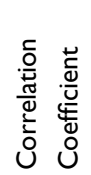 & 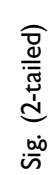 & 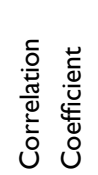 & 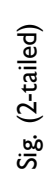 & 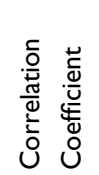 & 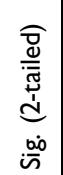 & 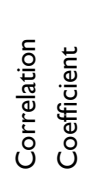 & 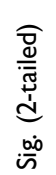 & 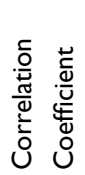 & 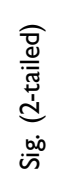 \\
\hline & 密 & & ڤొ & & 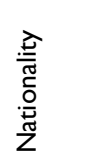 & & 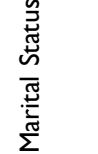 & & 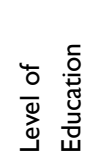 & & 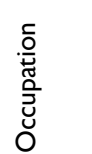 & & 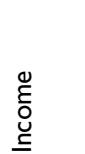 & \\
\hline
\end{tabular}




\begin{tabular}{|c|c|c|c|c|c|c|c|}
\hline 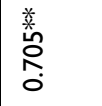 & $\stackrel{8}{\circ}$ & $\begin{array}{l}\text { 恙 } \\
\stackrel{0}{0} \\
0 \\
0\end{array}$ & 웅 & 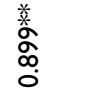 & ঃ & 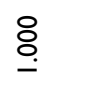 & \\
\hline 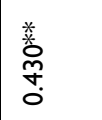 & : & $\begin{array}{l}\text { 美 } \\
\text { 学 } \\
0\end{array}$ & ঃ̊ & $\underset{-}{\stackrel{0}{0}}$ & & 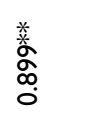 & ○. \\
\hline 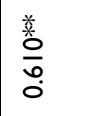 & $\stackrel{8}{\circ}$ & $\stackrel{\text { : }}{-}$ & & 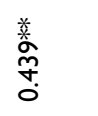 & ঃి & $\begin{array}{l}\text { 羔 } \\
\hat{\mathrm{h}} \\
0 \\
0\end{array}$ & @o \\
\hline ه্. & & $\frac{\text { 응 }}{0}$ & ঃ & $\begin{array}{l}\text { 幸 } \\
\text { fे. } \\
\stackrel{0}{0}\end{array}$ & ঃo & 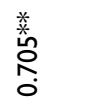 & ¿o \\
\hline 草 & $\stackrel{\bar{y}}{\mathrm{~N}}$ & ồ. & $\frac{\mathrm{m}}{0}$ & $\begin{array}{l}\text { 蓶 } \\
\text { i. }\end{array}$ & $\stackrel{\substack{\stackrel{n}{0} \\
0}}{0}$ & $\stackrel{\infty}{\circ}$ & $\begin{array}{c}\stackrel{0}{\infty} \\
\infty \\
\infty\end{array}$ \\
\hline \begin{tabular}{l}
0 \\
\hdashline \\
0 \\
$i$
\end{tabular} & $\frac{\stackrel{a}{m}}{0}$ & $\begin{array}{l}\stackrel{+}{0} \\
0 \\
0\end{array}$ & $\frac{\hat{f}}{0}$ & $\begin{array}{l}\mathscr{0} \\
\stackrel{i}{i}\end{array}$ & $\frac{\bar{\sigma}}{0}$ & $\begin{array}{l}\text { ô } \\
\text { o } \\
\text { i }\end{array}$ & హ. \\
\hline $\begin{array}{l}\tilde{T} \\
0 \\
0\end{array}$ & $\begin{array}{l}\hat{o} \\
\dot{o} \\
0 \\
0\end{array}$ & $\begin{array}{l}\text { J } \\
0\end{array}$ & 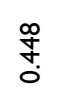 & $\begin{array}{l}\text { Pơ } \\
0 \\
0\end{array}$ & $\stackrel{\stackrel{n}{\sigma}}{\tilde{\sigma}}$ & 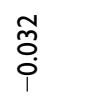 & 兽 \\
\hline ọ̊ & 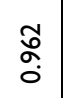 & $\begin{array}{l}\frac{m}{o} \\
\grave{i}\end{array}$ & $\begin{array}{c}\underset{\infty}{\infty} \\
\underset{0}{0} \\
0\end{array}$ & 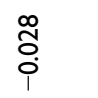 & 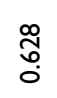 & 足 & $\bar{m}$ \\
\hline $\begin{array}{l}\text { 苮 } \\
\text { i. }\end{array}$ & 总 & 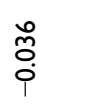 & 운 & $\begin{array}{l}\hat{\tilde{o}} \\
\stackrel{i}{i}\end{array}$ & 苂 & $\begin{array}{l}\stackrel{\mathscr{0}}{0} \\
\stackrel{0}{i}\end{array}$ & $\frac{8}{0}$ \\
\hline$\frac{\frac{*}{\dot{*}}}{i}$ & $\frac{\mathfrak{O}}{0}$ & $\frac{\stackrel{*}{m}}{i}$ & $\underset{\widetilde{0}}{\stackrel{0}{0}}$ & $\frac{\text { 善 }}{\frac{\alpha}{i}}$ & $\bar{\varnothing}$ & $\frac{\frac{*}{*}}{\frac{*}{\Lambda}}$ & ח̊. \\
\hline $\begin{array}{l}8 \\
\text { o. } \\
\text { i }\end{array}$ & $\frac{\tilde{N}}{0}$ & 훙 & 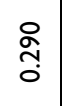 & $\frac{0}{0}$ & $\stackrel{\stackrel{8}{\circ}}{\stackrel{0}{0}}$ & $\begin{array}{l}\text { 응 } \\
\text { i }\end{array}$ & 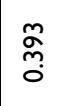 \\
\hline 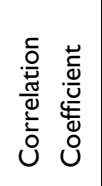 & 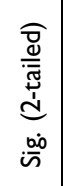 & 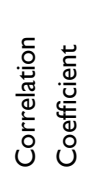 & 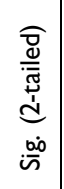 & 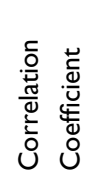 & 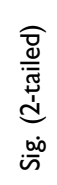 & 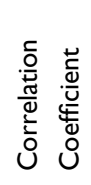 & 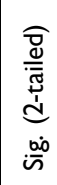 \\
\hline 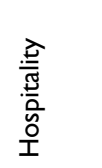 & & $\stackrel{\vec{\Phi}}{\stackrel{\vec{\omega}}{N}}$ & & 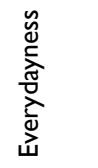 & & 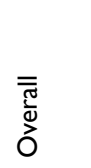 & \\
\hline
\end{tabular}

focuses on safety, everydayness, and hospitality. The climate of safety denotes on a safe environment that allows patients to receive medical treatment, whereas the climate of everydayness focuses on their positive thoughts about their illness or the health care setting where they are being treated. The climate of hospitality, on the other end, identifies the patients' expectations of being welcomed by healthcare professionals in a healthcare environment. ${ }^{26}$

In this study, the total mean score of PCQ-P was 73 (SD 9.9) and much higher than the total mean value of 59.7 (SD 11.5) reported in a study in China. ${ }^{29}$ This finding shows that most of the patients perceived the climate of care at the study hospital as highly person-centered. A high score indicates better quality of care for the patients by the health care workers in the tertiary hospital. This high level of quality care may be due to the availability of resources and better health policies implemented in Saudi Arabia. In contrast, another study in Norway reported a little higher PCQ-P mean scores of 86.5 (SD 11.4) using a 6-point Likert scale, unlike the 5-point scale selected for the current study. ${ }^{30}$ This score gap may be due to the scale difference and also reveals that there is room for further improvement of person-centered care.

The respondents in our study were highly satisfied with the climate of hospitality and safety. Similarly, residents in larger nursing homes in China also had a better perception of the climate of safety. ${ }^{29}$ Research shows that patients' perception is related to some factors including the availability and approachability of health care staff to care for them. ${ }^{31}$ Likewise, the climate of safety in any institution is associated with the skills and expertise of the available staff, their workload and the ability to identify and meet the patient's needs in a timely fashion. ${ }^{32}$

Another unexpected finding in the present study was patients' low perception of the climate of everydayness. In contrast, a US study conducted in a long-term care setting reported high mean scores for items 12 and 13 (4.21 \pm $1.67 \mathrm{SD}$, and $5.00 \pm 1.45 \mathrm{SD}$, respectively). ${ }^{25}$ Several personal characteristics like age, gender, occupation, area of residence, and the characteristics of health services were significantly associated with patients' perceptions of the PCC climate. Younger patients had a better perception of the overall climate of the PCC, hospitality, safety, and everydayness. This finding, however, differs from an earlier study correlating elderly patients with higher perceptions of PCC climate compared to their younger counterparts. ${ }^{33}$ In this study, age is more likely to be associated with the PCC climate, and younger residents 


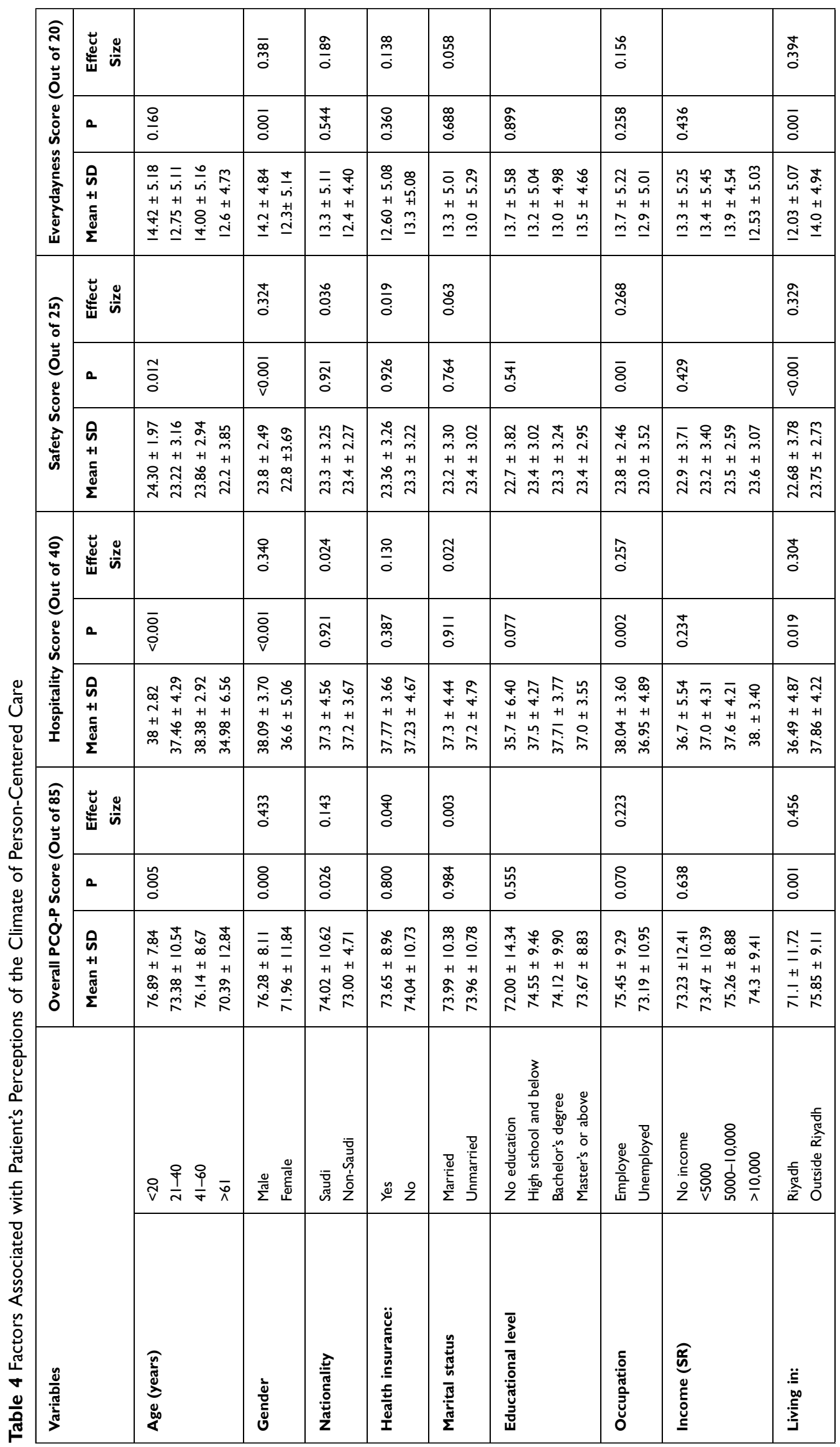




\begin{tabular}{|c|c|c|}
\hline రి & $\frac{n}{0}$ & $\stackrel{\widetilde{0}}{0}$ \\
\hline 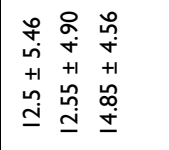 & 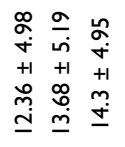 & 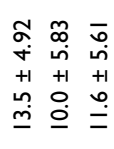 \\
\hline 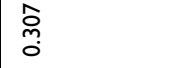 & 웅 & $\frac{0}{0}$ \\
\hline 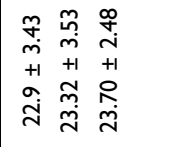 & 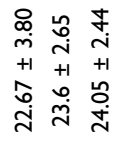 & 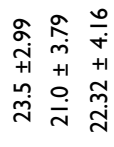 \\
\hline 员 & $\begin{array}{l}\text { No } \\
\text { O. }\end{array}$ & 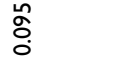 \\
\hline 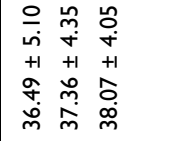 & 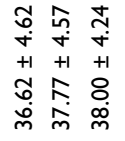 & 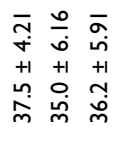 \\
\hline ర్ & $\stackrel{0}{0}$ & $\frac{0}{0}$ \\
\hline 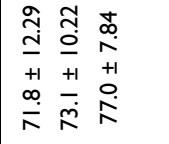 & 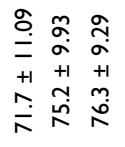 & 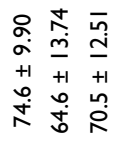 \\
\hline 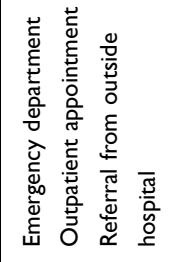 & $\bar{v} \stackrel{I}{\Lambda}$ & 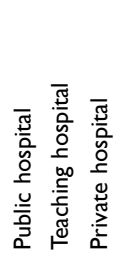 \\
\hline 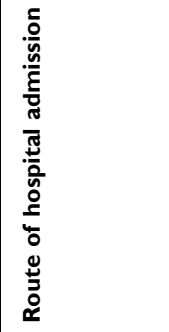 & 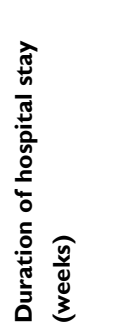 & 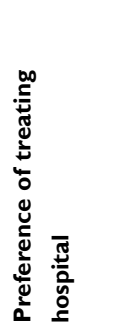 \\
\hline
\end{tabular}

were more satisfied with the person-centered care at the tertiary hospital in Saudi Arabia. While our findings contradict previous studies that overlook the relationship between age and patients' perceptions of the PCC, further research is warranted. Our study revealed that males had a better perception of the PCC climate than females, and this variation in perception might be due to their different expectations of the hospital environment and the availability of resources for care. ${ }^{29}$ Another significant factor associated with the climate of safety and hospitality was employment, but no significant relationship was observed with everydayness. This finding is consistent with previous studies on person-centered maternal care (PCMC) that showed that employed women had a better perception of the quality of care. ${ }^{34}$ Patients usually miss their own homes when living in long-term care facilities, yet they can adjust to the new environment. For example, establishing relations with caregivers in nursing homes may foster a smooth transition for the residents. ${ }^{35}$ Previous most early studies reported literature showed patients' literacy level reported among one of the determinants of their perception of PCC climate perceptions. However, education status was not a significant predictor of PCC in the current study. Although there was no significant association between literacy and the PCC climate, educated participants had a better perception than those with low literacy, which accords with previous studies.

\section{Strengths and Limitations}

This is the first study to assess person-centered care from the patients' perceptive in Arab healthcare settings. The study methods have been enhanced in terms of sample size as well as the type and statistical analysis. However, this study has some limitations. Firstly, the cross-sectional nature of the study is to be taken into consideration, as the cause and effect relation between predictors and perception cannot be established. Secondly, participants were recruited in only a single hospital, which may restrict the generalizability of the study. Sensitivity of the topic was another limitation as participant must be examined in the context of their health needs and previous interactions with healthcare providers.

\section{Conclusions}

This study assessed patients' perception of healthcare environments in a tertiary hospital in Saudi Arabia. Notably, the climate of care in the study setting seems highly person-centered. Perceptions are also significantly 
Table 5 Predictors of Patients' Perceptions of the Climate of Person-Centered Care

\begin{tabular}{|c|c|c|c|c|c|c|c|}
\hline \multirow[t]{3}{*}{ Variables } & \multirow[t]{3}{*}{ PCQ Scale } & \multicolumn{2}{|c|}{ Univariate Analysis } & \multirow[t]{3}{*}{$P$} & \multirow{2}{*}{\multicolumn{2}{|c|}{$\begin{array}{c}\text { Multivariate Analysis } \\
\text { Unstandardized Coefficients }\end{array}$}} & \multirow[t]{3}{*}{$\mathbf{P}$} \\
\hline & & \multicolumn{2}{|c|}{ Unstandardized Coefficients } & & & & \\
\hline & & B & SE & & B & SE & \\
\hline \multirow[t]{4}{*}{ Age } & Overall scale & -1.120 & 0.711 & 0.116 & - & - & - \\
\hline & Hospitality & -0.812 & 0.299 & 0.007 & -0.824 & 0.312 & 0.009 \\
\hline & Safety & -0.389 & 0.214 & 0.071 & - & - & - \\
\hline & Everydayness & -0.081 & 0.347 & 0.816 & - & - & - \\
\hline \multirow[t]{4}{*}{ Gender } & Overall & -0.610 & 0.245 & 0.013 & -0.452 & 0.257 & 0.078 \\
\hline & Hospitality & -0.571 & 0.255 & 0.025 & -0.451 & 0.264 & 0.088 \\
\hline & Safety & -0.516 & 0.265 & 0.051 & -0.387 & 0.273 & 0.157 \\
\hline & Everydayness & -0.823 & 0.240 & 0.001 & -0.723 & 0.248 & 0.004 \\
\hline \multirow[t]{4}{*}{ Nationality } & Overall & -0.918 & 0.657 & 0.162 & - & - & - \\
\hline & Hospitality & -0.388 & 0.658 & 0.555 & - & - & - \\
\hline & Safety & -0.594 & 0.659 & 0.367 & - & - & - \\
\hline & Everydayness & -0.127 & 0.644 & 0.844 & - & - & - \\
\hline \multirow[t]{4}{*}{ Income } & Overall & 0.507 & 0.531 & 0.340 & - & - & - \\
\hline & Hospitality & 0.466 & 0.226 & 0.040 & 0.501 & 0.262 & 0.057 \\
\hline & Safety & 0.268 & 0.162 & 0.099 & - & - & - \\
\hline & Everydayness & -0.194 & 0.258 & 0.452 & - & - & - \\
\hline \multirow[t]{4}{*}{ Health insurance } & Overall & 0.401 & 0.333 & 0.228 & - & - & - \\
\hline & Hospitality & 0.015 & 0.351 & 0.966 & - & - & - \\
\hline & Safety & -0.009 & 0.366 & 0.980 & - & - & - \\
\hline & Everydayness & -0.020 & 0.331 & 0.952 & - & - & - \\
\hline \multirow[t]{4}{*}{ Marital status } & Overall & 0.015 & $0.27 I$ & 0.957 & - & - & - \\
\hline & Hospitality & -0.094 & 0.277 & 0.733 & - & - & - \\
\hline & Safety & 0.035 & 0.292 & 0.904 & - & - & - \\
\hline & Everydayness & 0.006 & 0.264 & 0.983 & - & - & - \\
\hline \multirow[t]{4}{*}{ Educational level } & Overall & 0.607 & 0.825 & 0.462 & - & - & - \\
\hline & Hospitality & 0.627 & 0.351 & 0.075 & - & - & - \\
\hline & Safety & 0.212 & 0.250 & 0.396 & - & - & - \\
\hline & Everydayness & -0.218 & 0.399 & 0.586 & - & - & - \\
\hline \multirow[t]{4}{*}{ Occupation } & Overall & -0.213 & 0.260 & 0.413 & - & - & - \\
\hline & Hospitality & -0.320 & 0.274 & 0.243 & - & - & - \\
\hline & Safety & -0.372 & 0.287 & 0.195 & - & - & - \\
\hline & Everydayness & -0.308 & 0.252 & 0.221 & - & - & - \\
\hline
\end{tabular}

(Continued) 
Table 5 (Continued).

\begin{tabular}{|c|c|c|c|c|c|c|c|}
\hline \multirow[t]{3}{*}{ Variables } & \multirow[t]{3}{*}{ PCQ Scale } & \multicolumn{2}{|c|}{ Univariate Analysis } & \multirow[t]{3}{*}{$P$} & \multirow{2}{*}{\multicolumn{2}{|c|}{$\begin{array}{c}\text { Multivariate Analysis } \\
\text { Unstandardized Coefficients }\end{array}$}} & \multirow[t]{3}{*}{$\mathbf{P}$} \\
\hline & & \multicolumn{2}{|c|}{ Unstandardized Coefficients } & & & & \\
\hline & & B & SE & & B & SE & \\
\hline \multirow[t]{4}{*}{ Area of residency } & Overall & 0.737 & 0.246 & 0.003 & 0.529 & 0.267 & 0.047 \\
\hline & Hospitality & 0.764 & 0.254 & 0.003 & 0.558 & 0.271 & 0.040 \\
\hline & Safety & 0.467 & 0.262 & 0.075 & - & - & - \\
\hline & Everydayness & 0.693 & 0.242 & 0.004 & 0.444 & 0.261 & 0.089 \\
\hline \multirow[t]{4}{*}{ Route of hospital admission } & Overall & 0.421 & 0.159 & 0.008 & 0.309 & 0.171 & 0.071 \\
\hline & Hospitality & 0.385 & 0.164 & 0.019 & 0.277 & 0.176 & 0.116 \\
\hline & Safety & 0.124 & 0.167 & 0.457 & - & - & - \\
\hline & Everydayness & 0.357 & 0.153 & 0.020 & 0.270 & 0.165 & 0.101 \\
\hline \multirow[t]{4}{*}{ Duration of hospital stay } & Overall & 2.365 & 0.708 & 0.001 & 2.473 & 0.715 & 0.001 \\
\hline & Hospitality & 0.716 & 0.308 & 0.021 & 0.793 & 0.306 & 0.010 \\
\hline & Safety & 0.704 & 0.217 & 0.001 & 0.778 & 0.219 & 0.000 \\
\hline & Everydayness & 0.990 & 0.345 & 0.004 & 0.956 & 0.351 & 0.007 \\
\hline \multirow[t]{4}{*}{ Preference of treating hospital } & Overall & -0.422 & 0.173 & 0.015 & -0.384 & 0.179 & 0.032 \\
\hline & Hospitality & -0.163 & 0.173 & 0.349 & - & - & - \\
\hline & Safety & -0.437 & 0.172 & 0.011 & -0.417 & 0.176 & 0.018 \\
\hline & Everydayness & -0.231 & 0.172 & 0.179 & - & - & - \\
\hline
\end{tabular}

associated with patients' characteristics like age, gender, employment, and length of stay. And overall, to keep the momentum of PCC approach, whether in the current setting or a similar hospital, specialists and policymakers should work closely to adopt more person-centered healthcare systems and develop new and more efficient models of care. Moreover, future research is highly recommended to evaluate the PCQ-P in different health organizations in Arab hospitals context, exploring the staff perspective on PCC and how to integrate theories into practice in different settings for better outcomes.

\section{Implications}

Use of the PCQ-P would provide further evidence on which to base improvements in the quality of the healthcare system in Saudi Arabia and other Arab similar healthcare context. It would also shift the focus to the person-centered approach; encourage more healthcare providers in Saudi Arabia and Arab similar healthcare context to adopt PCC approaches and motivate hospital managers to integrate PCC into daily policy and practice of healthcare services.

\section{Abbreviations}

PCC, person (or patient)-centered care; PCQ-P, personcentered care climate questionnaire - patient version.

\section{Data Sharing Statement}

The datasets generated and/or analyzed during the current study are not publicly available due to the institutional rules and regulations but are available from the corresponding author on reasonable request.

\section{Ethics Approval and Consent to Participate}

The study proposal was reviewed by the research committee of the College of Public Health and Health Informatics for scientific approval, followed by further review by the Institutional Review Board (IRB) of King Abdullah 
International Medical Research Center. The approval was granted with the reference number SP16-079. IRB approval was also obtained from the Research Ethics Committee at the study hospital (reference: NP\&R/12/37). Patients meeting the inclusion criteria were asked to participate voluntarily in this study; those who verbally agreed to take part signed a written informed consent form that was kept in their file. The authors maintained and managed complete confidentiality, privacy and the freedom for participant to withdraw or stop their participation any time throughout and after the study. This study followed the recommendations of the International Conference on Harmonization for Good Clinical Practice (ICH-GCP) and in compliance with the Declaration of Helsinki.

\section{Acknowledgments}

We would like to thank the College of Public Health and Health Informatics at King Saud bin Abdulaziz University for Health Sciences for facilitating the process to conduct of this research project. Special appreciation to the study hospital staffs for their support and particularly nurses for their assistance with the collection of the data.

\section{Author Contributions}

All authors made substantial contributions to conception and design, acquisition of data, or analysis and interpretation of data; took part in drafting the article or revising it critically for important intellectual content; agreed to submit to the current journal; gave final approval of the version to be published; and agree to be accountable for all aspects of the work.

\section{Funding}

The author received no specific funding for this research.

\section{Disclosure}

The authors declare that they have no competing interests.

\section{References}

1. Institute of Medicine Committee on Quality of Health Care in, A. Crossing the Quality Chasm: A New Health System for the 21st Century. Washington (DC): National Academies Press (US) Copyright 2001 by the National Academy of Sciences. All rights reserved; 2001.

2. McCormack B, McCance T, Klopper H. Person-centred practice in nursing and health care: theory and practice; 2017. Available from: http://search.ebscohost.com/login.aspx?direct=true\&scope=site \&db= nlebk\&db=nlabk\&AN=1289475. Accessed March 26, 2021.

3. Kazemi A, Kajonius P. Assessing person-centred care: an item response theory approach. Int J Older People Nurs. 2020;e12352. doi:10.1111/opn.12352
4. Ekman I, Swedberg K, Taft C, et al. Person-centered care - ready for prime time. Eur $J$ Cardiovasc Nurs. 2011;10(4):248-251. doi:10.1016/j.ejcnurse.2011.06.008

5. Edvardsson D. Notes on person-centred care: what it is and what it is not. Nord $J$ Nurs Res. 2015;35(2):65-66. doi:10.1177/ 0107408315582296

6. Zhao J, Gao S, Wang J, Liu X, Hao Y. Differentiation between two healthcare concepts: person-centered and patient-centered care. Int J Nurs Sci. 2016;3(4):398-402. doi:10.1016/j.ijnss.2016.08.009

7. Robinson JH, Callister LC, Berry JA, Dearing KA. Patient-centered care and adherence: definitions and applications to improve outcomes. J Am Acad Nurse Pract. 2008;20(12):600-607. doi:10.1111/ j.1745-7599.2008.00360.x

8. DiGioia AM, Greenhouse PK. Care experience-based methodologies: performance improvement roadmap to value-driven health care. Clin Orthop Relat Res. 2012;470(4):1038-1045. doi:10.1007/s11999-0112051-3

9. Manias E, Bucknall T, Hutchinson A, Botti M, Allen J. Improving Documentation at Transitions of Care for Complex Patients. Sydney: ACSQHC; 2017.

10. Meterko M, Wright S, Lin H, Lowy E, Cleary PD. Mortality among patients with acute myocardial infarction: the influences of patientcentered care and evidence-based medicine. Health Serv Res. 2010;45(5p1):1188-1204. doi:10.1111/j.1475-6773.2010.01138.x

11. Allerby K, Goulding A, Ali L, Waern M. Striving for a more person-centered psychosis care: results of a hospital-based multi-professional educational intervention. BMC Psychiatry. 2020;20(1):523. doi:10.1186/s12888-020-02871-y

12. Brothers TD, Bonn M. Patient-centred care in opioid agonist treatment could improve outcomes. CMAJ. 2019;191(17):E460-E461. doi:10.1503/cmaj.190430

13. Buawangpong N, Pinyopornpanish K, Jiraporncharoen W, et al. Incorporating the patient-centered approach into clinical practice helps improve quality of care in cases of hypertension: a retrospective cohort study. BMC Fam Pract. 2020;21(1):108. doi:10.1186/s12875-020-01183-0

14. Bauman AE, Fardy HJ, Harris PG. Getting it right: why bother with patient-centred care? Med J Aust. 2003;179(5):253-256. doi:10.5694/ j.1326-5377.2003.tb05532.x

15. Pelzang R. Time to learn: understanding patient-centred care. $\mathrm{Br}$ J Nurs. 2010;19(14):912-917. doi:10.12968/bjon.2010.19.14.49050

16. Chua GP, Tan HK. A qualitative approach in determining the patient-centered information and supportive care needs of cancer patients in Singapore. BMJ Open. 2020;10(2):e034178. doi:10.1136/ bmjopen-2019-034178

17. Almoajel AM. Hospitalized patients' awareness of their rights in Saudi governmental hospital. Middle East J Sci Res. 2012;11 (3):329-335.

18. Al Asmri M, Almalki MJ, Fitzgerald G, Clark M. The public health care system and primary care services in Saudi Arabia: a system in transition. East Mediterr Health J. 2019;25.

19. Walston S, Al-Harbi Y, Al-Omar B. The changing face of healthcare in Saudi Arabia. Ann Saudi Med. 2008;28(4):243-250. doi:10.5144/ 0256-4947.2008.243

20. Ministry of Health's Vision Realization Office (VRO). (2019, 20 February). Healthcare Transformation Strategy. Available from: https://www.moh.gov.sa/en/Ministry/vro/Pages/HealthTransformation-Strategy.aspx. Accessed March 26, 2021.

21. Ahmad S, Singh J, Kamal MA, Shaikh ZM. Person-centered care design with reference to healthcare outcomes in Saudi Arabia: an overview. Am J Civ Eng Archit. 2020;8(3):91-96. doi:10.12691/ajcea-8-3-2

22. Aljuaid M, Elmontsri M, Edvardsson D, Rawaf S, Majeed A. Psychometric evaluation of the Arabic language person-centred climate questionnaire-staff version. $J$ Nurs Manag. 2018;26 (4):449-456. doi:10.1111/jonm. 12570 
23. Aljuaid M, Mannan F, Chaudhry Z, Rawaf S, Majeed A. Quality of care in university hospitals in Saudi Arabia: a systematic review. BMJ Open. 2016;6(2):e008988. doi:10.1136/bmjopen-2015-008988

24. Faul F, Erdfelder E, Lang A-G, Buchner A. G* Power 3: a flexible statistical power analysis program for the social, behavioral, and biomedical sciences. Behav Res Methods. 2007;39(2):175-191. doi:10.3758/BF03193146

25. Yoon JY, Roberts T, Grau B, Edvardsson D. Person-centered climate questionnaire-patient in English: a psychometric evaluation study in long-term care settings. Arch Gerontol Geriatr. 2015;61(1):81-87. doi:10.1016/j.archger.2015.03.010

26. Edvardsson D, Sandman PO, Rasmussen B. Swedish language person-centred climate questionnaire-patient version: construction and psychometric evaluation. $J$ Adv Nurs. 2008;63(3):302-309. doi:10.1111/j.1365-2648.2008.04709.x

27. Cai L, Ahlström G, Tang P, et al. Psychometric evaluation of the Chinese version of the Person-centred Climate Questionnaire - Staff version (PCQ-S). BMJ Open. 2017;7(8):e017250. doi:10.1136/bmjopen-2017-017250

28. Coe R. It's the effect size, stupid: what effect size is and why it is important; 2002.

29. Yang Y, Li H, Xiao LD, Zhang W, Xia M, Feng H. Resident and staff perspectives of person-centered climate in nursing homes: a cross-sectional study. BMC Geriatr. 2019;19(1):292. doi:10.1186/ s12877-019-1313-x
30. Bergland A, Hofoss D, Kirkevold M, Vassbø T, Edvardsson D. Person-centred ward climate as experienced by mentally lucid residents in long-term care facilities. J Clin Nurs. 2015;24(3-4):406-414. doi:10.1111/jocn. 12614

31. Edvardsson D, Sandman P-O, Rasmussen B. Construction and psychometric evaluation of the Swedish language Person-centred Climate Questionnaire-staff version. J Nurs Manag. 2009;17 (7):790-795. doi:10.1111/j.1365-2834.2009.01005.x

32. Chenoweth L, Forbes I, Fleming R, et al. PerCEN: a cluster randomized controlled trial of person-centered residential care and environment for people with dementia. Int Psychogeriatr. 2014;26 (7):1147-1160. doi:10.1017/S1041610214000398

33. Peck BM. Age-related differences in doctor-patient interaction and patient satisfaction. Curr Gerontol Geriatr Res. 2011;2011:1-10. doi:10.1155/2011/137492

34. Afulani PA, Sayi TS, Montagu D. Predictors of person-centered maternity care: the role of socioeconomic status, empowerment, and facility type. BMC Health Serv Res. 2018;18(1):360. doi:10.1186/ s12913-018-3183-X

35. Custers AF, Westerhof GJ, Kuin Y, Gerritsen DL, Riksen-Walraven JM. Relatedness, autonomy, and competence in the caring relationship: the perspective of nursing home residents. J Aging Stud. 2012;26(3):319-326. doi:10.1016/j.jaging.2012.02.005
Patient Preference and Adherence

\section{Publish your work in this journal}

Patient Preference and Adherence is an international, peer-reviewed, open access journal that focusing on the growing importance of patient preference and adherence throughout the therapeutic continuum. Patient satisfaction, acceptability, quality of life, compliance, persistence and their role in developing new therapeutic modalities and compounds to optimize clinical outcomes for existing disease

\section{Dovepress}

states are major areas of interest for the journal. This journal has been accepted for indexing on PubMed Central. The manuscript management system is completely online and includes a very quick and fair peer-review system, which is all easy to use. Visit http:// www.dovepress.com/testimonials.php to read real quotes from published authors. 